P94 Preliminary Efficacy of the 'Super Dads Super Kids' Web-Based Nutrition Education Program on Determinants of Behavior and Health Behaviors of Asian Indian Fathers and Their Elementary School-Age Children

Priya Krishnakumar, MS, Florida International University; Florence George, PhD, Florida International University; Catherine Coccia, $P h D, R D$, Florida International University, 11200 SW 8th St AHC5, Miami, FL, 33199

Background: Fathers' nutrition knowledge, attitudes and child feeding practices impact children's eating behavior and future weight. There is a need to target fathers in child nutrition education interventions, especially in ethnic groups like Asian Indians (AI).

Objective: The study aimed to examine the efficacy of a social cognitive theory (SCT) based online nutrition education program on determinants of behavior (knowledge, self-efficacy) and behavior (healthy eating, physical activity) in AI fathers and their children.

Study design, Settings, Participants: Ninety-eight AI fathers of 6-11-year-old children from various Indian communities in Florida were recruited to participate in the web-based nutrition education program. Fathers were asked to engage with the intervention for 6 weeks and topics included father's role in child health, ideal child feeding practices, healthy eating and physical activity. Validated survey questionnaires at baseline, post-intervention ( 6 weeks) and follow up (12 weeks) assessed participants' knowledge, child feeding, self-efficacy, selfregulation, diet and physical activity for fathers and their children.

Analysis: Descriptive statistics and Repeated Measures ANOVA were performed through SPSS v 26.

Results: Majority of AI fathers were between 35-44 years (75.5\%). There was a statistically significant increase on the AI fathers' nutrition knowledge (F $=120.93, P<$ $0.01)$, self-efficacy $(\mathrm{F}=19.980, P<0.01)$, self-regulation $(\mathrm{F}=10.387, P<0.01)$, overall diet quality $(\mathrm{F}=12.257$, $P<0.01)$, physical activity $(\mathrm{F}=32.790, P<0.01)$ and $\mathrm{a}$ reduction in restrictive feeding practices $(\mathrm{F}=5.683, P=$ $0.004)$ through the intervention. Improvements were also noted in father reported child fruit and vegetable intake $(\mathrm{F}=30.821, P<0.01)$, total fat consumed/day (F $=4.040, P=0.020)$ and physical activity minutes $(\mathrm{F}=$ 15.018, $P<0.01)$. Post hoc analyses revealed significant changes between baseline and post-intervention for all 9 outcomes and post-intervention and follow up for AI fathers' diet related self-efficacy and self-regulation scores.

Conclusion: Web-based interventions are effective to improve behavioral determinants and behavior in AI fathers' and their children. Future studies should consider diverse AI father groups for childhood obesity prevention.

Funding: None.

\section{P95 Process and Outcome Evaluation of the LSU AgCenter SNAP-Ed Program Using Microsoft Dynamics 365}

Matthew Greene,LDN, RD, MSPH, mgreene@agcenter.lsu. edu, Louisiana State University (LSU) Agriculture Center, 110 LSU Union Square, Baton Rouge, LA, 70805; Andrew Garcia, BS, Louisiana State University Agriculture Center; William Lee, BS, Louisiana State University Agriculture Center; Denise Holston, PhD, LDN, RD, Louisiana State University Agriculture Center

Background: Supplemental Nutrition Assistance Program Education (SNAP-Ed) encourages healthier eating through education and policy, systems, and environmental (PSE) changes to support access to healthier foods. Collecting and managing data for process and outcome evaluations is challenging for SNAP-Ed implementing agencies (IAs) with variation in direct education and PSE change projects implemented. Several IAs have used the Program Evaluation and Reporting System (PEARS) to gather and manage evaluation data, but this may be cost prohibitive for IAs with smaller budgets. The LSU AgCenter Information Technology (IT) department adapted Microsoft Dynamics 365 to the evaluation needs of the LSU AgCenter SNAP-Ed program.

Objective: To conduct a process evaluation of direct education and PSE change work conducted by the LSU AgCenter SNAP-Ed program in Federal Fiscal Year 2019-2020 using the Microsoft Dynamics 365 Application.

Study Design, Setting, Participants: LSU AgCenter IT staff adapted Dynamics to collect the data necessary to report against priority indicators in the SNAP-Ed evaluation framework. IT staff created separate reporting modules for individual direct education contacts, groups of direct education contacts, community partnerships, community coalitions, and PSE changes. SNAP-Ed frontline staff were trained on the reporting system in a series of hands-on, in-person meetings held prior to March 2020.

Measurable Outcomes/Analysis: LSU AgCenter staff report direct education contacts, partnerships, coalitions, and PSE changes made in the Microsoft Dynamics application. Data can be disaggregated according to demographic information, location, and implementing staff member, enabling data to be used in performance evaluations and analyses of equity in program access.

Results: Feedback about the reporting system was overwhelmingly positive. The system collected data necessary for reporting to state and federal funding agencies. Over the course of FY2020, LSU AgCenter established 502 partnerships, made 6,473 direct education contacts, and 118 PSE changes.

Conclusion: The Microsoft Dynamics 365 Application was easily adapted to the evaluation needs of the LSU AgCenter SNAP-Ed program. Process and outcome evaluation data indicated that the LSU AgCenter met its goals for FY2020.

Funding: Supplemental Nutrition Assistance Program Education. 